\title{
Dynamic Characteristic Analysis of Electric Actuator for 1 kV/3.2 kA Air Circuit Breaker Based on the Three-link Structure
}

\author{
Seung-Min Lee ${ }^{\dagger}$ Jong-Ho Kang*, Sang-yup Kwak*, Rae-Eun Kim* and Hyun-Kyo Jung*
}

\begin{abstract}
In the present paper, a new type of electrical actuator, an electromagnetic force driving actuator (EMFA), applicable to air circuit breaker is developed and analyzed. Transient analysis is performed to obtain the dynamic characteristics of EMFA. The distribution of static magnetic flux is obtained using the finite element method. The coupled problems of electrics and mechanics governing equations are solved using the time-difference method. According to the interception rate of each contactor, investigation of the contactor spring load condition is conducted and applied to the threelink system. Comparisons of the dynamic characteristics of the three-link simulation and experimental data are performed.
\end{abstract}

Keywords: Air circuit breaker, Electromagnetic force driving actuator, Finite element method Time difference method

\section{Introduction}

The low-voltage air circuit breaker (ACB) has been the backbone of industrial power systems since the 1940s. It has been challenged by competitive devices such as fuses, molded case breakers, and, more recently, insulated case breakers; however, it remains superior [1].

In ACB, the contacts of ACB consist of mechanical operating parts and arc contacts. To overcome mechanical contact loads containing holding force and arc repulsive force, to fulfill the required stroke length, as well as the average velocity of an extinguishable arc area at contacts, typical mechanics such as spring, pneumatic, and hydraulic mechanism actuators are generally used in this field. These conventional actuators have high energy density to overcome such loads; however, they are composed of many parts. Thus, they have many linked sections and low tolerance, which decrease the reliability and endurance of switchgear, and which require periodic repairs and replacement of parts.

To overcome these problems, interest in electric actuators, including solenoid, motor, and permanent magnetic actuators (PMAs) has recently been renewed [2]. PMA has the remarkable merits of having fewer components, having higher reliability, being maintenancefree, and having more easily controllable electrical parameters [3]. However, owing to its immense magnetic loss at large stroke length, the utility of PMA is limited in

\footnotetext{
$\dagger \quad$ Corresponding Author: School of Electrical Engineering and Computer Science, Seoul National University, Korea. (mayu2000@snu.ac.kr)

* School of Electrical Engineering and Computer Science, Seoul

National University, Korea. (hkjung@snu.ac.kr)

Received: April 28, 2009; Accepted: January 5, 2011
}

the high-voltage class. In addition, PMA is very large compare to spring actuator, so it is not applicable to the ACB.

As a result, a new type of electromagnetic force driving actuator (EMFA) is proposed and developed [4]. EMFA has high performance that can meet a long-stroke length as well as high load of contactor. It is smaller compared to PMA, and is operated by Lorentz force, which is a magnetic force between the permanent magnet and inserted iron core. EMFA is able to neglect magnetic loss at air gap, and it has prominent power with a long stroke length.

By adopting a three-link structure, the contact load of interrupter can be better mitigated compared to the direct link system. The stroke length and average velocity of the interrupter are changeable, and can convert the ratio of the three-link structure.

\section{General Structure of EMFA and the Three-link Structure}

\subsection{Structure of EMFA}

Consider an elemental structure of the EMFA shown in Fig. 1. The specifications of the proposal analysis of EMFA for $1 \mathrm{kV} / 3.2 \mathrm{kA} \mathrm{ACB}$ are listed in Table 1. EMFA consists of five parts: a moving coil, an inserted moving iron core, a static core, permanent magnets, and transmission rods. The molded moving part consists of bidirectional coil, inserted core, and transmission rod. The bidirectional coil and inserted core are held in place by the magnetic force of the permanent magnets. The transmission rod is a coupling 
device that transmits opening or closing operations to the three-link system.
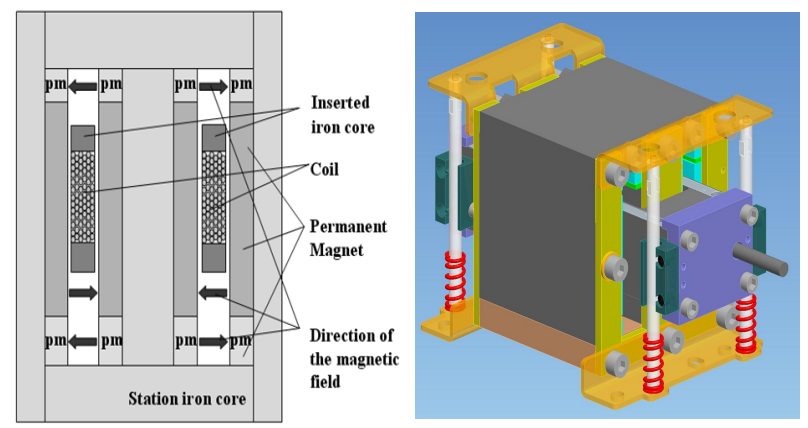

Fig. 1. Basic structure of EMFA

The DC voltage source is connected to an energy banker (capacitor) through a charger. The Lorenz force, which is the magnetic flux cross current density, affects the moving part directly. Its direction can be translated from a full bridge IGBT switching. It is also able to control the force and velocity of the moving part directly by controlling the current and inductance of the coils appropriately [5]-[6].

\subsection{Three-link structure linked with EMFA}

The electromagnetic force is transmitted from EMFA to contact through the three-link rigid frame. At this time, it has nonlinear dynamic characteristics of displacement between EMFA and contact. Fig. 2 shows the three-link structure linked with EMFA. The shape of this nonlinear curve is somewhat different from the displacements of the actuator (X) and contacts (Y). In the simulation, the lengths of frames (L1, L2, and L3) are 90.05, 51.98, and $84 \mathrm{~mm}$ each and the initial angle of $\theta 1$ and $\theta 2$ are 7.76 and 51.77. The nonlinear stroke curve enhances the high-speed capability of the arc extinguishment at the arc region, and reduces the condensed contact load, enabling a short range to spread widely.

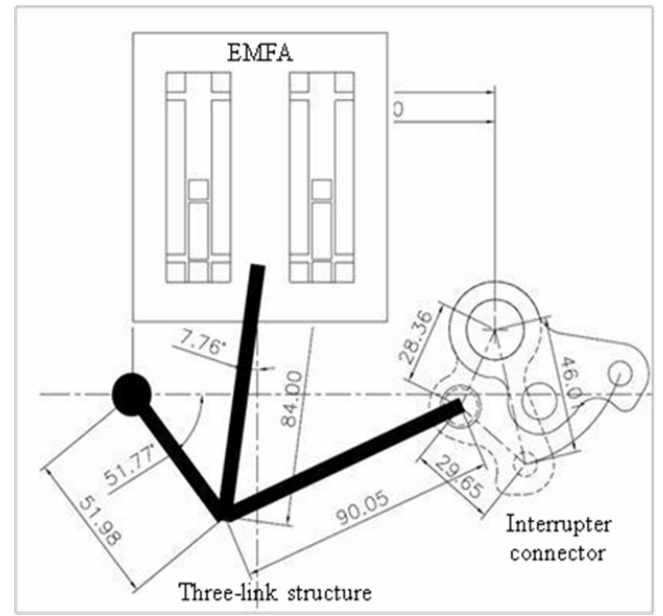

Fig. 2. Three-link structure linked with EMFA

\section{Numerical Analysis}

To design EMFA and predict its performance as an actuator for the ACB, transient analysis is necessary. The static magnetic field analysis method based on electromagnetic finite element method (FEM) and the coupled problems of electrics and mechanics for analysis of dynamic characteristics based on time-difference method (TDM) are explained in detail. The results of the analysis have led to the development of an electromagnetically, as well as mechanically, optimal design.

\subsection{FEM and TDM analysis}

In this section, some fundamental static magnetic equations are described, as presented in the below equations. Using Maxwell's equation and magnetic vector potential, Eq. (1) is given:

$$
\nabla \times\left(\frac{1}{\mu} \nabla \times \vec{A}\right)=\overrightarrow{J_{0}}+\overrightarrow{J_{e}}+\left(\nabla \times \frac{1}{\mu_{r}} \overrightarrow{M_{r}}\right)
$$

where $J_{0}\left(\mathrm{~A} / \mathrm{m}^{2}\right)$ is the current density, $J_{e}\left(\mathrm{~A} / \mathrm{m}^{2}\right)$ is the eddy current density, $M(\mathrm{~A} / \mathrm{m})$ is the amount of magnetization, $M_{r}(\mathrm{~A} / \mathrm{m})$ is the amount of residual magnetism, $A\left(\mathrm{~Wb} / \mathrm{m}^{2}\right)$ is the magnetic vector potential, and $\mu(\mathrm{H} / \mathrm{m})$ is the permeability.

To solve simply the magnetic flux, $A$ is used, as derived from Eq. (1):

$$
\Phi=\oint \vec{A} d l
$$

where $\Phi(\mathrm{Wb})$ is the magnetic flux and $l(\mathrm{~m})$ is the total coil length.

The electromagnetic force is divided into two basic types: the holding and the operating force [Eqs. (3) and (4)]. The holding force is generated by a permanent magnet without external current [Eq. (3)]:

$$
\vec{F}^{\text {hold }}=\iint_{s}\left\{\frac{1}{\mu_{0}}(\vec{B} \cdot \vec{n}) \vec{B}-\frac{1}{2 \mu_{0}} B^{2} \cdot \vec{n}\right\} d s
$$

where $F^{\text {hold }}(\mathrm{N})$ is the electromagnetic holding force, $s\left(\mathrm{~m}^{2}\right)$ is the area of the outer surface around the iron core of the moving part, and $n$ is the unit vector of the normal direction along the surface.

The external current is excited; therefore, the electromagnetic operating force is generated by the magnetic density cross the current density, as presented in Eq. (4) [4]:

$$
\vec{F}^{\text {operate }}=q(\vec{E}+\vec{v} \times \vec{B})
$$


where $F^{\text {operate }}(\mathrm{N})$ is the Lorenz force, which is the magnetic force during the moving part's traveling; $q$ (C) is the electric charge; and $v(\mathrm{~m} / \mathrm{s})$ is the velocity vector.

In the coupled problems of electrics and mechanics, external current flows into the moving coil from the capacitor, and its value changes as time passes. Therefore, transient analysis is necessary in TDM.

Generally, an electrical equation includes back electromotive force and inductance, which are formulated as follows:

$$
V=\left(I_{0}+d i\right)+N\left(\frac{\partial \varphi}{\partial i}\right)\left(\frac{d i}{d t}\right)+N\left(\frac{\partial \varphi}{\partial z}\right)\left(\frac{d z}{d t}\right) .
$$

where $V(\mathrm{~V})$ is the voltage of the capacitor, $I_{0}(\mathrm{~A})$ is the initial value of the exerted current, $d i(\mathrm{~A})$ is the variation of exerted current, $d t(\mathrm{~s})$ is the unit of the time variation, and $N$ (turn) is the turns of coils [4]. The second term of the right side is the transformer voltage and the third is the speed voltage. At the very beginning, the holding force is greater than the operating force. Although the current rises into the coil, the moving part does not move. During this period, the third term goes to zero.

The basic kinematical equation is as follows:

$$
m\left(\frac{d^{2} z}{d t^{2}}+g\right)=F^{\text {hold }}+F^{\text {operate }}+F^{\text {spring }}
$$

where $m(\mathrm{~kg})$ the moving part which are bidirectional coil and inserted core mass, which includes the moving parts of the actuator in addition to the mass of the transmission rod and circuit interrupter; $z(\mathrm{~m})$ is the operating length; and $F^{\text {spring }}(\mathrm{N})$ is the contact load of the interrupter.

\subsection{Three-link Analysis}

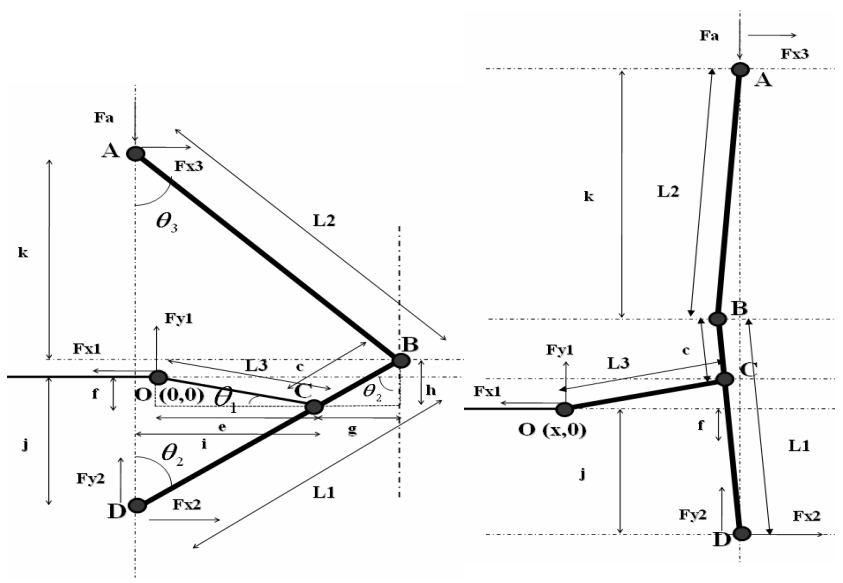

Fig. 3. Equilibrium on three-link frame diagram

This link frame is composed of three free-body frames $\left(L_{1}, L_{2}\right.$, and $\left.L_{3}\right)$ connected at Nodes $A, B, C$, and $D$, as shown in Fig. 3.
Node $D$ is a principle rotary fixed axis, which is a rotation within an interior angle $\left(\theta_{2}\right)$ and the main frame $L_{1}$, which is linked between Nodes $D$ and $B$. The frame $L_{3}$ is linked with EMFA and Node $C$, which is a rotary connector positing at between Nodes $D$ and $B$. It transmits the electromagnetic force to the frame $L_{l}$ and Node $B$, a point of contact with frame $L_{1}$ and frame $L_{2}$. Finally, electromagnetic force is transmitted through Node B to reach the contacts. The The angles $\theta_{2}$ and $\theta_{3}$ have a restricted operation angle in a variety of ways, and lengths of the frames $L_{1}$ and $L_{2}$ are decided by contact stroke length. In the same way, the length of frame $L_{3}$ and the angle $\theta_{1}$ are determined by the stroke of EMFA and the magnitude of the transmitted compressed contact force.

EMFA operates as follows. Node $O$, which moves toward the direction of the X-axis, linked frame $L_{3}$, and $L_{3}$ frame is connected at Node $C$. Curvilinear coordinates in a rate of $\left\{\left(L_{2}-c\right) / L_{2}\right\}$ are drawn at Node $B$. Through Node $B$, the transmitted force of EMFA drives Node $A$, which is located at the end of the frame $L_{2}$, to move upward for contact. Following the displacement of EMFA $(d x)$ at the point of Node $O$, the contact moving distance $(d y)$ is presented as follows:

$$
\begin{gathered}
\Delta y=h^{\prime}-f^{\prime}+k^{\prime}-h+f-k \\
h^{\prime}=c \cdot \cos \left(\theta_{1}^{\prime}\right), f^{\prime}=L_{3} \cdot \sin \left(\theta_{1}^{\prime}\right), \\
\theta_{1}^{\prime}=\sin ^{-1}\left\{\frac{B-\left(L_{1}^{\prime}+i^{\prime}\right)^{2}}{L_{3}} \cdot \cos \left(\theta_{2}^{\prime}\right)\right. \\
\theta_{2}^{\prime}=\sin ^{-1}\left\{\frac{m n-\sqrt{\left(m^{2} n^{2}\right)-\left(m^{2}-1\right)\left(n^{2}+1\right)}}{n^{2}+1}\right\} \\
g^{\prime}=c \cdot \sin \left(\theta_{2}^{\prime}\right), i^{\prime}=\left(L_{2}-c\right) \cdot \cos \left(\theta_{2}^{\prime}\right) \\
m=\frac{E}{2 \cdot L_{1} \cdot c}, n=\frac{A}{B}
\end{gathered}
$$

The static equilibrium of force on the free-body diagram (FBD) is as follows:

$$
f x_{1}=\frac{(j+h+k) \cdot L_{2} \cdot \sin \left(\theta_{2}\right) \cdot \cos \left(\theta_{1}\right)}{k \cdot\left(L_{2}-c\right) \cdot \cos \left(\theta_{1}-\theta_{2}\right)} \cdot F_{a}
$$

where $F_{\mathrm{a}}(\mathrm{N})$ is the contact force at Node $\mathrm{A}$ and $F x_{1}$ is the transmitted force to the EMFA. As a result, the characteristic of contact load can be mitigated by its nonlinear feature, as shown in Fig. 4. The nonlinear curve is the spring force measured at the actuator, whereas the linear line is circulated numerically at the position of the interrupter. Conversing condensed contact force at the last stroke to the middle section, the highest spring force dwindles by approximately $30 \%$. This shows that in the three-link system, a smaller actuator can be used for the interrupter. 


\section{Dynamic Characteristics of Experiment Results}

In Fig. 5, at the very beginning, the inclination of the actuator-interrupter stroke is very sharp; however, it becomes nearly horizontal at the last stroke section. At the former stroke section, its nonlinear curve facilitates the fulfillment of the required average velocity of the interrupter in the arc area at the opening operation. The velocity of the interrupter is very slow at the last stroke section. This suggests that EMFA greatly reduces chattering at the closing event, and diminishes abrasion on the interrupter during operation. The moving iron core of EMFA is held in place by the magnetic force of the permanent magnet. Therefore, EMFA is a latch-free device. When opening or closing, the holding force becomes an

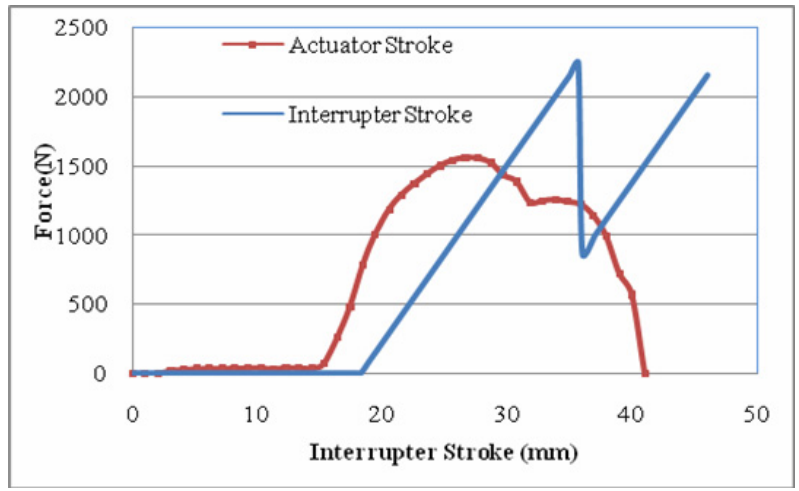

Fig. 4. Spring force of interrupter

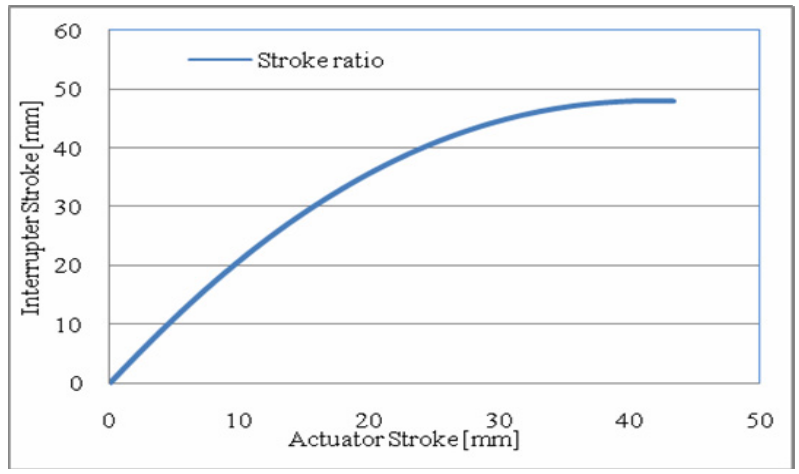

Fig. 5. Actuator-interrupter stroke ratio

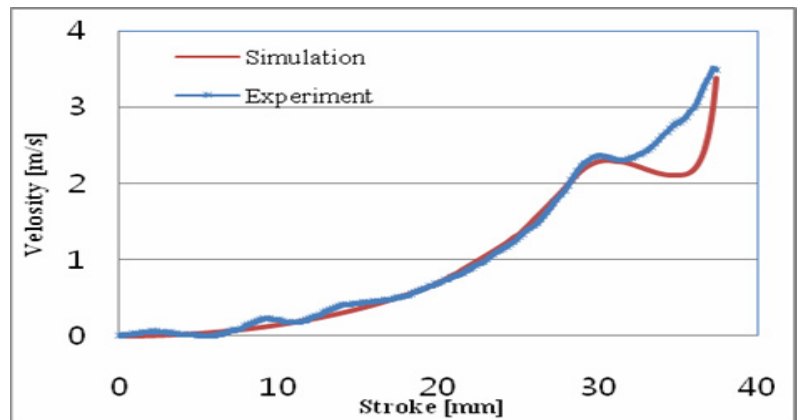

Fig. 6. Simulation and experiment velocity data on actuator at closing operation obstacle to the required velocity of the interrupter and interception time. As shown in Fig. 2, the axis angle of the driving force and impetus is exactly $90^{\circ}$. As for this threelink structure, the holding force can be decreased desperately when we design EMFA. Fig. 6 shows the dynamic characteristics of experiment and simulation results. These experimental data can be obtained using a streak camera. The time-velocity curve of the simulation results is very smooth compared to that of the experimental results. Some differences in the charging time section before EMFA moves are evident. In the experimental data, from 0.1 to $18 \mathrm{~ms}$, the interrupter that connects the threelink structure experienced significant tremor. The prototype of the three-link system appeared to have a degree of tolerance. The alignments of the three-link structure did not match in this experiment; therefore, the force of EMFA was not transmitted fully to the interrupter at that time region. The force of the actuator hit the comparatively heavy load of the three-link, elastic energy reflected in EMFA. In the last stroke part, some differences between the experiment and the simulation also became evident. In that stroke area, the interrupter load spring affected the dynamic characteristic of EMFA. The interrupter connected to the three-link part appeared to have some tolerance; thus, it did not conduct the force directly to EMFA. As a result, the former area of velocity of the actuator was slowly estimated. In simulation results, frictional force was not conserved, and the overall pattern of simulation data matched well with the experimental data.

\section{Conclusion}

In the present paper, EMFA for $1 \mathrm{kV} / 3.2 \mathrm{kA} \mathrm{ACB}$ combined with the three-link system is presented and is proven to be a good actuator for ACB. Through the analysis of dynamic characteristics, which combined FEM and time different method, EMFA was developed with predictable mechanical stability in the primary design stage. In addition, using the three-link system, EMFA was proven to be a more reliable actuator for ACB. The simulation results were verified against experimental data.

\section{References}

[1] W. S. Jackson, Vice President "The air circuit breaker 50 years of technology" Digital Object Identifier 10.1109/TEXCON. 1997

[2] E. Dullni, "A Vacuum Circuit-breaker with Permanent Magnetic Actuator for Frequent Operations", IEEE 18thInternational Symposium on Discharges and Electrical Insulation in VacuumEindhoven, pp. 688-691, 1998.

[3] Xiong, Guangyu, and Yamada, "Dynamic analysis of a cylindrical moving-core linear oscillator actuator", 
Linear Drives for Industry Applications 95 Nagasaki, JAPAN, pp. 405-408, 1999.

[4] J.H. Kang, and H. K. Jung, "Development and Characteristic Analysis of New type Actuator, Electro Magnetic driven Force Actuator applicable to High Voltage Circuit Breaker" The Fifth International Symposium on Linear Drives for Industry Applications, 25-28 September 2005, pp. 383 386.

[5] W. Kim, S. Y. Kwak, J. Lee, D. Choi, H. K. Jung, K. Seong, and S. Hahn, "Design of HTS magnets for a 600 kJ SMES," IEEE Trans. on Appl. Supercond., vol. 16, no. 2, pp. 620-623, May 2006.

[6] Ma Shaohua, and Wang jimei, "Research and Design of Permanent Magnetic Actuator for High Voltage Vacuum Circuit Breaker", IEEE XXth international Symposium on Discharges and Electrical Insulation in Vacuum, pp. 487-490, 2002

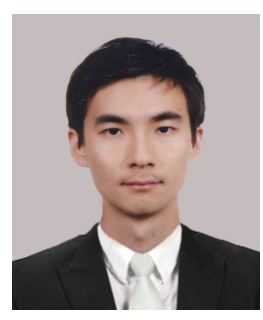

Seung-Min Lee received his B.S in electrical engineering from Seoul National University. His research interests are numerical analysis and the design of electrical machines.

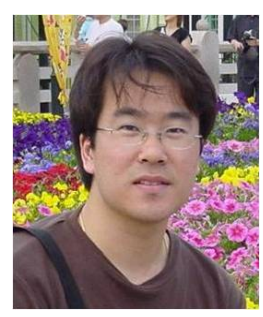

Jong-Ho Kang received his B.S degree in electrical engineering from Chung-Ang University. His research interests are numerical analysis and design of electrical-machines.

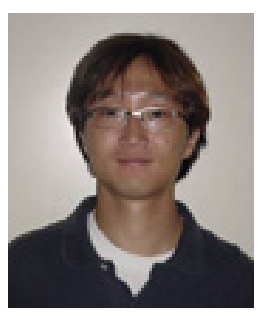

Sang-yup Kwak received his B.S in electrical engineering from Seoul National University. His research interests are numerical analysis and design of electrical machines.

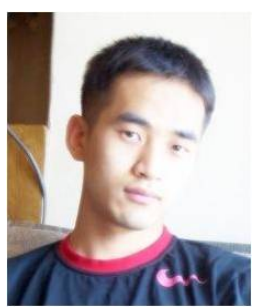

Rae-Eun Kim received his B.S in electrical engineering from Seoul National University. His research interests are numerical analysis and the design of electrical machines

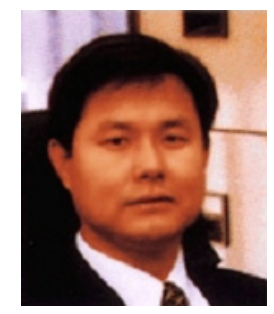

Hyun-Kyo Jung received his $\mathrm{PhD}$ in electrical engineering from Seoul National University. His research interests are electric machinery, EM waves, circuit breakers, and so on. 